\title{
Performance Comparison of Sleep Mode Operations in IEEE 802.16e Terminals ${ }^{\star}$
}

\author{
Youn-Hee Han ${ }^{1}$, Sung-Gi Min ${ }^{2}$, and Dongwon Jeong ${ }^{3}$ \\ ${ }^{1}$ School of Internet-Media, Korea University of Technology and Education, \\ Cheonan, Korea \\ yhhan@kut.ac.kr \\ ${ }^{2}$ Department of Computer Science and Engineering at Korea University, \\ Seoul, Korea \\ sgmin@korea.ac.kr \\ ${ }^{3}$ Department of Informatics and Statics, Kunsan National University, \\ Gunsan, Korea \\ djeong@kunsan.ac.kr
}

\begin{abstract}
IEEE 802.16e Task Group has developed an IEEE 802.16 amendment supporting mobile terminals such as PDAs, phones, and laptops. The energy efficiency design is an important research issue for mobile terminals due to limited battery power of them. In this paper, we study the energy efficiency design of IEEE 802.16e. For the purpose of power efficient operation, there are two sleep mode operations related to normal data traffic in the current IEEE 802.16e specification. We propose analytical models of the two sleep mode operations and conduct their performance comparison in terms of the power consumption and the frame response time.
\end{abstract}

Keywords: IEEE 802.16e, Sleep Mode.

\section{Introduction}

Broadband wireless access systems use base stations (BSs) to provide broadband multimedia services to business or homes as an alternative to wired 'last-mile' access links that use fiber, cable, or digital subscriber lines. A promising advance of such systems is the IEEE 802.16 standard, which provides fixed-wireless access between the subscriber station and the Internet service provider (ISP) 12. The IEEE 802.16 standardization is also expected to support quality of service (QoS) for real time application such as voice over IP. For such QoS support, it defines four different scheduling services: Unsolicited Grant Service (UGS), Real-Time Variable Rate (RT-VR), Non-Real Time Variable Rate (NRT-VR), and Best Effort (BE) services [3].

Whereas the existing IEEE 802.16 standards address fixed wireless applications only, IEEE 802.16e standard [4] aims to serve the needs of fixed, nomadic,

\footnotetext{
* This work was supported by the Korea Research Foundation Grant funded by the Korean Government (MOEHRD) [KRF-2006-331-D00539].
} 
and fully mobile networks. It enhances the original standard with mobility so that Mobile Subscribe Stations (MSSs) can move during services. It adds mobility support to the original standard so that MSSs can move while receiving services. Mobility of MSSs leads the power consumption aware design, which is one of the primary objectives due to limited computation power of MSSs 5 . In order to get the solution with the lowest power consumption, optimization over all aspects of system implementation must be employed, including the algorithms, architectures, circuit design, and manufacturing technology. In this paper, we are interested in reducing power consumption for IEEE 802.16e MSSs in terms of MAC algorithms.

In the current IEEE 802.16e specification, three sleep mode operations, named Power Saving Class (PSC) 1, 2, and 3, are defined for the purpose of power efficient operations. Among the three PSCs, PSC 1 and 2 are related to data traffic, while PSC 3 serves for management procedure such as periodic raging. While PSC 1 serves data traffic carried in NRT-VR or BE connection, PSC 2 serves UGS or RT-VR data flow. In [6] and [7, the authors proposed the novel models to investigate the energy consumption in IEEE 802.16e. However, they evaluated only PSC 1 and did not perform any analysis about PSC 2 and PSC 3. In this paper, we propose analytical models of PSC 2 as well as PSC 1 and conduct their performance comparison in terms of their power consumption and frame response time. From this analytical study, we can get the features of the sleep mode operations in detail and obtain some hints about the way to determine right values of the system parameters used in sleep mode operation.

This paper is organized as follows. In Section 2, we represent the current operational rules of the two sleep mode operations, PSC 1 and 2, based on the IEEE 802.16e specification. In Section 3, we give an analysis about PSC 1 and 2. Section 4 conducts the performance evaluation and reveals the features of the operations, and concluding remarks are given in Section 5.

\section{Sleep Mode Operations in IEEE 802.16}

An IEEE 802.16 MSS has two modes: wakeup mode and sleep mode. The sleep mode is defined for the purpose of power efficient operations. Before entering the sleep mode, an MSS in the wake mode sends the sleep request message to a serving BS. After receiving the sleep response message which notifies whether the sleep request of the MSS is approved or not, the MSS can enter the sleep mode. In the sleep response message, the following relevant parameters are included: the start frame number for first sleep window, the minimum sleep interval, the maximum sleep interval, and the listening interval. The intervals are presented as units of MAC frames. After getting approval, the MSS goes into the sleep mode, gets sleep for an interval, and then wakes up to check whether there are frames for it. If there are frames for the MSS, it goes to the wakeup mode. Otherwise, the MSS is still in the sleep mode and gets sleep for another interval. The MSS keeps performing the above procedure until it goes to the wakeup mode. 
In the first power saving mode, PSC 1, sleep intervals are defined as follows. At the first sleep interval, the minimum sleep interval is used. Then each sleep interval is doubled until the maximum sleep interval is reached, and then the sleep interval keeps the maximum sleep interval. After each sleep interval, the MSS temporarily wakes up a short interval, called listening interval, to listen the traffic indication message broadcasted from the serving BS, and the message includes information about MSSs to whom the BS has frames waited. Furthermore, the MSS can terminate the sleep mode if there is an out-going frame, mostly because of the user's manual interaction.

IEEE 802.16 supports four QoS supports: UGS, RT-VR, NRT-VR, and BE. Each scheduling service is characterized by a mandatory parameter set of QoS parameters, which is adjusted to best describe the guarantees required by the applications that the scheduling service is designed for. UGS is designed to support real-tme applications with strict delay requirements. UGS is free from any contention of frame transmission. The BS provides fixed size data grants at periodic intervals to the UGS flows. UGS can be used for constant bit-rate (CBR) service. RT-VR and NRT-VR flows are polled through the bandwidth request polling. RT-VR is designed to support real-time applications with less stringent delay requirements. The supported applications may generate variable-size data packets at periodic intervals, such as MPEG video and VoIP with silence suppression. RT-VR flows is prevented from using any contention requests. While RT-VR can be used for real-time service, NRT-VR can be used for non-real time service such as bandwidth-intensive file transfer. The main difference between RT-VR and NRT-VR is that NRT-VR connections are reserved a minimum amount of bandwidth. Finally, BE is for applications with no rate or delay requirements.

PSC 1's behavior well fits the behavior of demand created by random (or bursty) IP traffic, like WEB browsing. Such traffic typically is carried in NRTVR or BE connection. But demand created by UGS and in some cases of RT-VR connections may have different pattern. For UGS (and RT-VR) flow, PSC 2 is recommended to be used. PSC 2 defines the different sleep interval from PSC 1's one. At the first sleep interval, the minimum sleep interval is used. However, the size of all subsequent sleep intervals is the same as the initial one. As a result, the definition of sleep mode for an MSS has been extended.

\section{Analytical Models}

In this paper, we are interested in the sleep mode operation affected by frames delivered from network to MSSs. For the model of incoming traffic flow, we use a simple train model. A frame train is defined in [8] as a burst of frames arriving from the same source and heading to the same destination. If the spacing between two frames exceeds some inter-train time, they are said to belong to different trains. For both PSC 1 and 2, we assume that the train arrival processes follow a Poisson distribution with the same rate $\lambda$. That is, UGS (or RT-VR) traffic and NRT-VR (or BE) traffic arrive at the same rate on the average. Accordingly, the inter-train times follow an exponential distribution with the mean $1 / \lambda$ (unit time). 


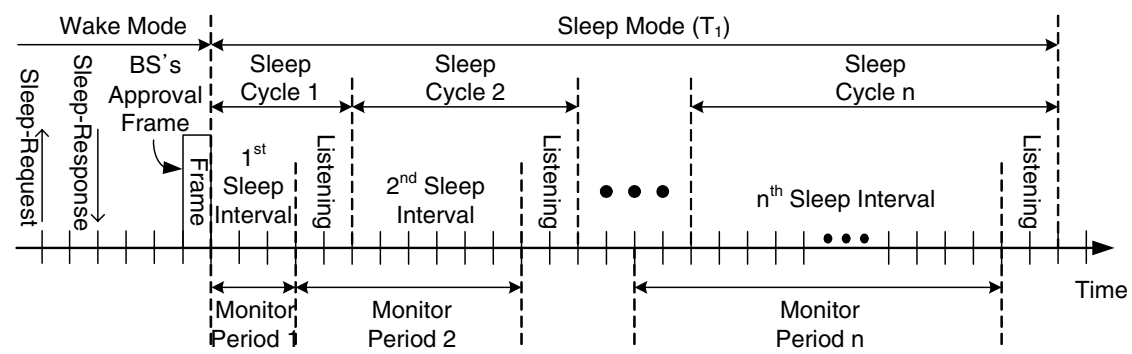

(a) Power Saving Class 1

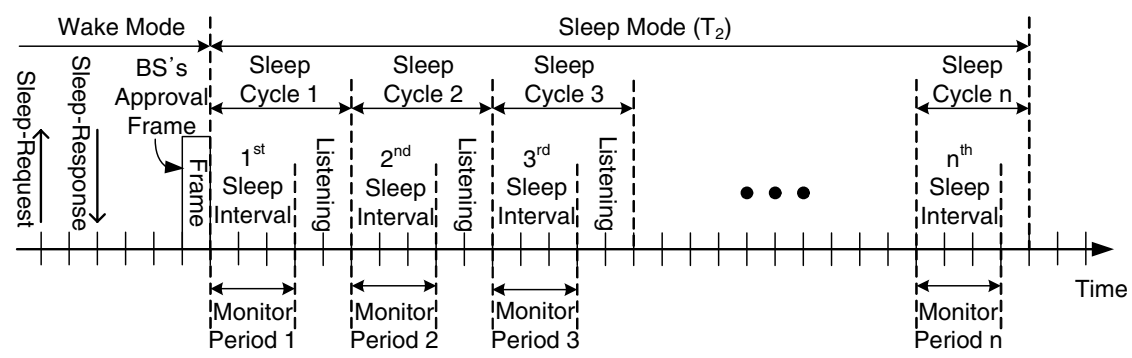

(b) Power Saving Class 2

Fig. 1. Sleep Mode Operations of IEEE 802.16e

We will use $T_{1}$ and $T_{2}$ to respectively denote the sleep mode interval of PSC 1 and 2. A sleep mode interval includes one or more sleep cycles, which are illustrated in Fig. 1 (a) and (b). A sleep cycle includes a sleep interval and a listening interval. It is assumed that the same listening interval $L$ is used for both PSC 1 and 2. Let $t_{1}^{1}$ and $t_{2}^{1}$ denote the initial sleep intervals of PSC 1 and PSC 2, respectively.

Let $t_{1}^{i}$ denote the length of MSS's $i$-th sleep interval in PSC 1 . The maximum sleep interval is $t_{\max }\left(=2^{M} t_{1}^{1}\right)$ where $M$ is the number of increments when the sleep window size reaches $t_{\max }$. Then, $t_{1}^{i}$ is defined as follows:

$$
t_{1}^{i}= \begin{cases}2^{i-1} t_{1}^{1} & \text { if } 1 \leq i<M \\ t_{\max } & \text { if } i \geq M .\end{cases}
$$

Similarly, let $t_{2}^{i}$ denote the length of MSS's $i$-th sleep interval in PSC 2 . For every $i$, according to the specification of PSC 2, it is simply defined as follows:

$$
t_{2}^{i}=t_{2}^{1}
$$

In PSC $1, L$ is only used for sending the traffic indication message to MSSs and enabling MSS synchronization with BS. So, the transmission of traffic arrived during a sleep interval begins at the end of the following listening interval $L$. When there is no traffic addressed to an MSS during a sleep interval and frames arrive in the listening interval immediately following the sleep interval, these 
packets are buffered and the traffic indication message for these packets will be notified at the next listening interval. In PSC 2, as opposite to PSC 1 , the transmission of traffic arrived during a sleep interval begins at the end of the sleep interval. That is, during $L$, an MSS can receive (or send) any frames from (or to) the serving BS without any exchange of notification. Based on these assumptions, we also define the monitor period to be the time interval when the frames arrived at BS should be buffered and released after $i$-th sleep cycle. For PSC 1 and PSC 2, the monitor periods are denoted by $s_{1}^{i}$ and $s_{2}^{i}$, respectively. They are given by

$$
\begin{aligned}
& s_{1}^{i}= \begin{cases}t_{1}^{1} & \text { if } i=1 \\
L+t_{1}^{i} & \text { if } i \geq 2 .\end{cases} \\
& s_{2}^{i}=t_{2}^{i}=t_{2}^{1} .
\end{aligned}
$$

Let $n_{1}$ denote the number of sleep cycles before an MSS goes to the wake mode in PSC 1. In PSC 1, the current sleep mode turns to the wake mode when a frame train arrives during a monitor period. Let $e_{1}^{i}$ denote the event that there is the train arrival for an MSS at a BS during the monitor period $i$ of PSC 1 . Then, we have

$$
\begin{gathered}
\operatorname{Pr}\left(e_{1}^{i}=\text { true }\right)=1-e^{-\lambda s_{1}^{i}} . \\
\operatorname{Pr}\left(n_{1}=1\right)=\operatorname{Pr}\left(e_{1}^{1}=\text { true }\right)=1-e^{-\lambda s_{1}^{1}}=1-e^{-\lambda t_{1}^{1}} .
\end{gathered}
$$

and for $k \geq 2$

$$
\begin{aligned}
\operatorname{Pr}\left(n_{1}=k\right) & =\operatorname{Pr}\left(e_{1}^{1}=\text { false } \wedge e_{1}^{2}=\text { false } \wedge \wedge e_{1}^{k-1}=\text { false } \wedge e_{1}^{k}=\text { true }\right) \\
& =\prod_{i=1}^{k-1} \operatorname{Pr}\left(e_{1}^{i}=\text { false }\right) \cdot \operatorname{Pr}\left(e_{1}^{k}=\text { true }\right) \\
& \left.=e^{-\lambda \sum_{i=1}^{k-1} s_{1}^{i}} \cdot\left(1-e^{-\lambda s_{1}^{k}}\right)=e^{-\lambda\left((k-1) L+\sum_{i=1}^{k-1} t_{1}^{i}\right.}\right) \cdot\left(1-e^{-\lambda\left(L+t_{1}^{k}\right)}\right) \cdot(
\end{aligned}
$$

Let $n_{2}$ denote the number of sleep cycles before MSS goes to the wake mode of PSC 2. Unlike PSC 1, PSC 2's sleep mode can turn to the wake mode when a frame train arrives during a listening interval or when it arrives during a monitor period. Similarly with Equations (5)-(7), we have

$$
\begin{aligned}
\operatorname{Pr}\left(n_{2}=k\right) & =e^{-\lambda(k-1)\left(t_{2}^{k-1}+L\right)} \cdot\left(1-e^{-\lambda\left(t_{2}^{k}+L\right)}\right) \\
& =e^{-\lambda(k-1)\left(t_{2}^{1}+L\right)} \cdot\left(1-e^{-\lambda\left(t_{2}^{1}+L\right)}\right) .
\end{aligned}
$$

Let $P_{S}$ and $P_{L}$ denote the power consumption units per a unit of time in the sleep interval and the listening interval, respectively. Assuming $P C_{1}$ denote the power consumption during the PSC 1's sleep mode interval. Then, assuming that $E[\cdot]$ stands the average function, we can get the average power consumption $E\left[P C_{1}\right]$ as follows: 


$$
\begin{aligned}
E\left[P C_{1}\right]= & \sum_{k=1}^{\infty} \operatorname{Pr}\left(n_{1}=k\right) \cdot \sum_{i=1}^{k}\left(t_{1}^{i} P_{S}+L P_{L}\right) \\
= & \left(1-e^{-\lambda t_{1}^{1}}\right)\left(t_{1}^{1} P_{S}+L P_{L}\right) \\
& +\sum_{k=2}^{\infty} e^{-\lambda\left((k-1) L+\sum_{i=1}^{k-1} t_{1}^{i}\right)} \cdot\left(1-e^{-\lambda\left(L+t_{1}^{k}\right)}\right) \cdot \sum_{i=1}^{k}\left(t_{1}^{i} P_{S}+L P_{L}\right) .
\end{aligned}
$$

Let $R_{1}$ denote the response time of a train's first frame in PSC 1 . In our paper, like [6], the response time represents the amount of time required for data packets to be delivered to an MSS after they are buffered at a BS. Since train arrivals follow Poisson distribution, the arrival events are random observers [9] to the sleep intervals. Therefore, we have

$$
\begin{aligned}
E\left[R_{1}\right] & =\sum_{k=1}^{\infty} \operatorname{Pr}\left(n_{1}=k\right) \cdot \frac{s_{1}^{k}}{2} \\
& =\left(1-e^{-\lambda t_{1}^{1}}\right) \frac{t_{1}^{1}}{2}+\sum_{k=2}^{\infty} e^{-\lambda\left((k-1) L+\sum_{i=1}^{k-1} t_{1}^{i}\right)} \cdot\left(1-e^{-\lambda s_{1}^{k}}\right) \frac{s_{1}^{k}}{2} .
\end{aligned}
$$

The PSC 2's last sleep cycle can finish without the full listening interval since PSC 2 allows MSS and BS to exchange frames even during the listening interval. For getting $E\left[T_{2}\right]$ exactly, therefore, it is required to model the last sleep cycles minutely. Again, the last sleep cycle can end up with a sleep interval plus the one among the following two cases: 1) no listening interval if a frame train arrives during the last monitor period, and 2) an interval time $(<L)$ when a frame train arrives after the last monitor interval. In second case, for simplicity, we just assume that the arrival time of a frame train is distributed uniformly on the listening interval $L$. So, we can get the average power consumption $E\left[P C_{2}\right]$ during the PSC 2's sleep mode interval as follows:

$$
\begin{aligned}
E\left[P C_{2}\right]= & \sum_{k=1}^{\infty} \operatorname{Pr}\left(n_{2}=k\right) \cdot\left(\sum_{i=1}^{k-1}\left(t_{2}^{i} P_{S}+L P_{L}\right)+\left(1-e^{-\lambda s_{2}^{k}}\right) \cdot t_{2}^{k} P_{S}+e^{-\lambda s_{2}^{k}} \cdot\left\lceil\frac{L}{2}\right\rceil P_{L}\right) \\
= & \left(1-e^{-\lambda\left(t_{2}^{1}+L\right)}\right) \cdot \sum_{k=1}^{\infty} e^{-\lambda(k-1)\left(t_{2}^{1}+L\right)} \\
& \cdot\left((k-1)\left(t_{2}^{1} P_{S}+L P_{L}\right)+\left(1-e^{-\lambda t_{2}^{1}}\right) \cdot t_{2}^{1} P_{S}+e^{-\lambda t_{2}^{1}} \cdot\left\lceil\frac{L}{2}\right\rceil T_{L}\right)
\end{aligned}
$$

Let $R_{2}$ denote the response time of a train's first frame in case of PSC 2. If the first frame arrives when MSS is in a sleep interval, it should be buffered at the serving BS until the sleep interval comes to end. Such buffering makes the response time long. Otherwise (the first frame arrives when MSS is in a listening interval), it does not cause the response time to be extended. Therefore, we have

$$
E\left[R_{2}\right]=\left(1-e^{-\lambda t_{2}^{1}}\right) \cdot \frac{t_{2}^{1}}{2}+e^{-\lambda t_{2}^{1}} \cdot 0=\left(1-e^{-\lambda t_{2}^{1}}\right) \cdot \frac{t_{2}^{1}}{2} .
$$




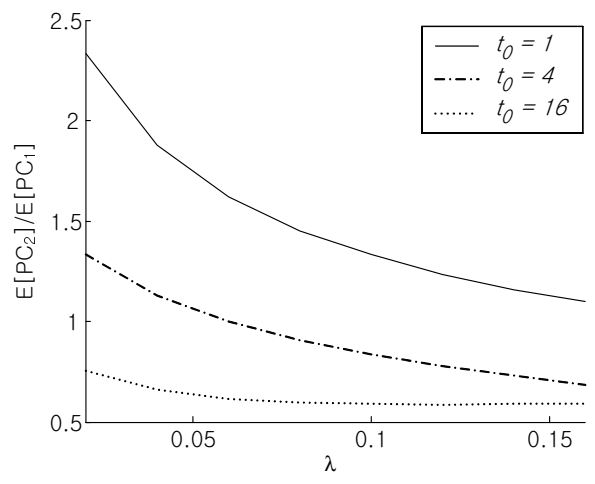

(a) $E\left[P C_{2}\right] / E\left[P C_{1}\right]$ with regard to $\lambda$

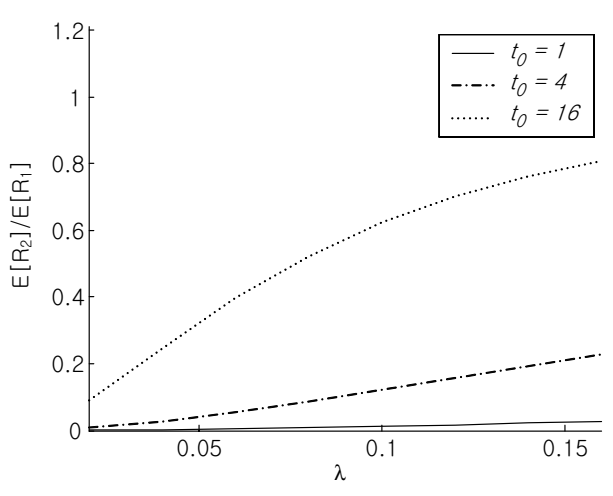

(b) $E\left[R_{2}\right] / E\left[R_{1}\right]$ with regard to $\lambda$

Fig. 2. Results of Performance Evaluation

\section{Performance Evaluation}

In the previous work [7, the author also conducted simulation for PSC 1's sleep mode operation and validated his analytic results with the simulation results. In this paper, we did not conduct a simulation for verification of our analysis. But, we assert that our analytical results are also reliable since our analytical method is based on [7]'s one and we extend its research results. For our analysis, the following default parameters are used: $L=1, t_{\max }=1024$ (thus, $M=10$ ), $P_{S}=1$, and $P_{L}=10$. We also assume that two initial sleep intervals of PSC 1 and PSC 2 are set to the same value $t_{0}$ (that is, $t_{1}^{1}=t_{2}^{1}=t_{0}$ ).

An interesting result is shown from Fig. 2 (a). Since PSC 1 has mostly longer sleep intervals than PSC 2, at a first glance, it might also seem that PSC 1's energy efficiency is generally better than PSC 2's one. However, PSC 2's energy efficiency is always better than PSC 1's one if $t_{0}$ is high (e.g., $t_{0}=16$ ). Such energy gain is furthermore almost insensitive to $\lambda$ (the values of $P C_{2} / P C_{1}$ are distributed around 0.6 in case of $t_{0}=16$ ). These are justified by the fact that PSC 1's listening interval is only used for sending the traffic indication message to MSSs, while an MSS can exchange a frame with its serving BS during PSC 2's listening interval. So, the last sleep cycle of PSC 2 can finish without the full listening interval. If initial sleep interval is so long, a frame train is very likely to arrive in the first or second sleep cycle.

Of a practical interest is the case of $t_{0}=4$. We can see PSC 2's energy efficiency is just similar to PSC 1 's one when $t_{0}=4$. If $\lambda>0.06$, it is even better than PSC 1 's one. It is because that a high $\lambda$ is also likely to make a frame train arrive in the first or second sleep cycle. If $\lambda$ is very high, therefore, a very small value of $t_{0}$ (e.g., $\left.t_{0}=1\right)$ is a not bad choice, which also guarantees a very fast frame response time.

Fig. 2 (b) also provides the comparison about the response time of a train's first frame. It shows that the dependence of the response time from the values 
of $\lambda$ is marginal if the values of $t_{0}$ are small (e.g., $t_{0}=1$ or 4 ). We also see that PSC 2's reduction of the response time is remarkably enormous with such small values of $t_{0}$. When $t_{0}=4$, PSC 2 achieves more than $80 \%$ of response time reduction. When $t_{0}=1$, the reduction is distributed even between $95 \%$ and $99 \%$. This result is of fundamental reason for PSC 2 to be developed for UGS (or RT-VR) traffic.

\section{Conclusions}

In this paper, we studied the details of two sleep mode operations, PSC 1 and PSC 2, presented by the recent specification of IEEE 802.16e. We also presented analytical models of PSC 1 and PSC 2 to compare them in terms of the two performance metrics:The power consumption $\left(E\left[P C_{1}\right]\right.$ and $\left.E\left[P C_{2}\right]\right)$ and the response time of a train's first frame $\left(E\left[R_{1}\right]\right.$ and $\left.E\left[R_{2}\right]\right)$.

This paper has focused how much PSC 2 reduces the response time of a train's first frame. We also studied how much it sacrifices the energy efficiency for reducing the response time. From our analysis results, we can conclude that the small values of $t_{0}$ (e.g., $t_{0}=1$ or 4 ) can make PSC 2 reduce the response time greatly (up to $80 \% \sim 99 \%$ ) at not much expense of the energy efficiency. Moreover, if $\lambda$ is very high, very small values of $t_{0}$ does not much sacrifice the energy efficiency while preserving a very low frame response time.

\section{References}

1. Std. 802.16-2004, I.: IEEE 802.16 Local and Metropolitan Area Networks - Part 16: Air Interface for Fixed Broadband Wireless Access Systems (2004)

2. Eklund, C., Marks, R.B., Stanwood, K.L., Wang, S.: IEEE Standard 802.16: A Technical Overview of The WirelessMAN Air Interface for Broadband Wireless Access. IEEE Communications Magazine 40(6) (June 2002) 98-107

3. Chu, G., Wang, D., Mei, S.: A QoS Architecture for The MAC Protocol of IEEE 802.16 BWA System. In: IEEE International Communications, Circuits and Systems and West Sino Expositions. Volume 1. (July 2002) 435-439

4. Std 802.16e 2005, I., Std 802.16-2004/Cor 1-2005, I.: IEEE Standard for Local and Metropolitan Area Networks Part 16: Air Interface for Fixed and Mobile Broadband Wireless Access Systems Amendment 2 (February 2006)

5. Xiao, Y., Chen, C.L.P., Kinateder, K.J.: An Optimal Power Saving Scheme for Mobile Handsets. In: Sixth IEEE Symposium on Computers and Communications (ISCC'01). (July 2001) 192-197

6. Seo, J.B., Lee, S.Q., Park, N.H., Lee, H.W., Cho, C.H.: Performance Analysis of Sleep Mode Operation in IEEE 802.16e. In: IEEE 60th Vehicular Technology Conference (VTC2004-Fall). Volume 2. (Sept. 2004) 1169-1173

7. Xiao, Y.: Energy Saving Mechanism in The IEEE 802.16e Wireless MAN. IEEE Communications Letters 9(7) (2005) 595-597

8. Jain, R., Routhier, S.A.: Packet Trains - Measurements and A New Model for Computer Network Traffic. IEEE Journal on Selected Areas in Communications 4(6) (1986) 986-995

9. Ross, S.M.: Stochastic Processes. Wiley (1995) 